\title{
Review of: "Association of maternal sleep before and during pregnancy with sleep and developmental problems in 1-year-old infants"
}

Akiyoshi SHIMURA

Potential competing interests: The author(s) declared that no potential competing interests exist.

This study has investigated and showed the association between maternal pre and during pregnant sleep habits and infants development.

<Major concern>

The viewpoint is very unique and the importance of sleep duration is suggested if the message of this study is true, however, there is a serious doubt of spurious correlation.

This study does not prove that the maternal short sleep duration affects infants' sleep disturbance or development.

Sleep duration and sleep quality (especially in slow-wave sleep) are strongly affected by their age and sleep hygiene.

Age is an important confounding. As people get older, they sleep less. Maternal (also paternal) age is a risk factor for the various health problems of infants including developmental disorders, which cause sleep disturbance and behavioral problems. This study did not control their age in the multivariate analyses or examine the effect of maternal age on the infants' outcome.

Sleep hygiene also could be a serious confounding. Poor sleepers will have a poorer sleep environment and it may affect their infants. The light exposure at night, poor light at daytime, noisy environment, taking caffeine, use of alcohol or tobacco, irregular lifestyle rhythm... they affect both of mothers' and infants' sleep.

$<$ Strong suggestion from the reviewer $>$

1) To examine those confounding and control them.

2) To disclose any statistical variable which guarantees the reliability of the analyses such as Nagelkerke $\mathrm{R}^{\wedge} 2, \mathrm{AIC}$, or PPV.

3) If the authors could not use the data about sleep hygiene, discuss it carefully.

$<$ minor issues $>$

Table 1

- To understand the characteristics of the parent population, it'd be better to add the column which 
indicates the distribution of the variables in the total participants (ex. How many boys in total are there? How many smokers are there in total?)

- Were there significant differences among each sleep duration category? It will be desirable to perform any assessments ( $\chi^{\wedge} 2$, ANOVA, or any other comparison). 